\title{
Online maritime health information: an overview of the situation
}

\author{
Matthieu J. Guitton ${ }^{1,2}$ \\ ${ }^{1}$ Faculty of Medicine, Laval University, Quebec City, QC, Canada \\ ${ }^{2}$ Institut Universitaire en Santé Mentale de Québec, Quebec City, QC, Canada
}

\begin{abstract}
Background: Due to their working conditions, seafarers often don't benefit from the same medical coverage than the onshore population. Therefore, seafarers and their relatives often need to locate health information by themselves. While the rise of the Internet has drastically transformed the way people can gather information, the availability of specific maritime health information online still need to be evaluated scientifically. We aim here to document of the characteristic of maritime health-related online information. Materials and methods: A web survey was performed, articulated on two complementary analyses. First, an overall analysis of websites related to maritime health compared to websites related to two other health areas relevant for the general population (dental health and otorhinolaryngology) used as control. Second, an analysis of the understandability and actionability of a series of Wikipedia articles related to pathologies relevant for seafarers using the Patient Education Materials Assessment Tool (PEMAT).

Results: Online resources associated with maritime health were sparse and difficult to locate. When compared to other medical fields, maritime health websites were extremely poor in displaying useful information for seafarers. Available online resources regarding specific diseases affecting seafarers were mainly not adapted for a general audience and scored poorly both in terms of understandability and of actionability. Conclusions: This study provides a general overview of the degree of adaption of online material related to maritime health to seafarers' potential needs. Considerably more efforts need to be made in order to provide controlled online materials to answer the health information needs of the seafarers and their relatives.
\end{abstract}

(Int Marit Health 2015; 66, 3: 139-144)

Key words: medical information, PEMAT, online survey, readability, seafarer health

\section{INTRODUCTION}

Due to their unique working conditions - long periods of isolation, constant travelling, exposure to dangerous environmental conditions, occupational hazards, etc. - seafarers have always been a vulnerable population regarding health issues. While the situation is better in passenger ships, with the presence of medical personnel and the availability of specialised equipment are combined with shorter durations far from the shores, the vast majority of seafarers - whether in commercial ships, in fisheries industries, or in offshore platforms - are experiencing the full disadvantages of sea travels [1, 2]. However, when at sea, seafarers have access to less medical facilities and advices than the population ashore. Therefore, seafarers and their relatives often need to gather health information by themselves.

In the last few decades, the rise of the new technologies of information, and particularly the democratisation of the Internet, has revolutionised the way information gets aggregated and accessed [3]. Indeed, the population increasingly turns to online spaces to locate relevant information, even for subjects as complex as science or medicine [3]. However, while the characteristics in terms of readability, understandability, and actionability of the information 
available online for various medical conditions are being the target of intensive scrutiny (see for instance the case of otorhinolaryngology [4-6]), the online resources related to maritime health have elicited so far less interest [7]. Therefore, this study aimed to offer a documented overview of the availability of online maritime health information for seafarers or their relatives.

\section{MATERIALS AND METHODS}

\section{WEB SURVEY AND WEBSITES ANALYSIS}

Since English is the most widely used language for information gathering on the Internet, from search engines to Wikipedia articles $[8,9]$, we focused the present survey on website written in English language. Three categories of websites were considered. The first category was made of websites related to maritime health. The two other categories served as control for maritime health sites, and consisted of two other medical or health-related specialties: dental health, and otorhinolaryngology/head and neck surgery. For each of these three categories, four different websites were selected (Table 1). Websites on maritime health were selected as those being the most easily accessible after a simply search using the Google search engine, i.e., the first websites (highest rankings in the search) explicitly labelled as related to maritime health appearing when using the search keywords "maritime health" or "maritime medicine" (Table 1). For the two control categories, the websites of four national associations were selected from countries having English as a first and native language (Table 1). All of the websites were last accessed the same day (June 22, 2015) for a final evaluation.

In addition, four articles were selected on the English version of Wikipedia, on diseases selected among those known to have a high prevalence amongst seafarers, or specifically related to aeronautical travel. The four diseases selected were malaria, heart disease, depression, and mal de debarquement (also known as debarkation sickness, Table 1). These articles were considered as representative of the resources available through the online encyclopaedia. Articles were last accessed the same day (June 22, 2015).

\section{WEBSITES ANALYSIS}

For each website, the following parameters were recorded: 1) the presence of health-related information pages targeted to the population (as opposed to information pages related to health professionals); 2) whether these pages were accessible directly from the homepage or if

Table 1. List of the websites surveyed in the present study

\begin{tabular}{|c|c|}
\hline Name & Web address \\
\hline \multicolumn{2}{|l|}{ Maritime health } \\
\hline International Maritime Health Association & http://www.imha.net/ \\
\hline International Seafarers' Welfare and Assistance Network & https://www.seafarerswelfare.org/ \\
\hline Seafarer Health - World Shipping Council & $\begin{array}{l}\text { http://www.worldshipping.org/industry-issues/safety/ } \\
\text { seafarer-health }\end{array}$ \\
\hline International Transport Worker's Federation (ITF) Seafarers & http://www.itfseafarers.org/ \\
\hline \multicolumn{2}{|l|}{ Otorhinolaryngology/head and neck surgery } \\
\hline American Academy of Otorhinolaryngology - Head and Neck Surgery & https://www.entnet.org/ \\
\hline Canadian Society of Otorhinolaryngology - Head and Neck Surgery & http://www.entcanada.org/ \\
\hline The Australian Society of Otorhinolaryngology - Head and Neck Surgery & http://www.asohns.org.au/ \\
\hline The New Zealand Society of Otorhinolaryngology, Head and Neck Surgery & http://www.orl.org.nz/ \\
\hline \multicolumn{2}{|l|}{ Dental health } \\
\hline American Dental Association & http://www.ada.org/en/ \\
\hline Canadian Dental Association & http://www.cda-adc.ca/en/index.asp \\
\hline Australian Dental Association & http://www.ada.org.au/ \\
\hline New Zealand Dental Association & https://www.nzda.org.nz/pub/ \\
\hline \multicolumn{2}{|l|}{ Wikipedia articles } \\
\hline Malaria & http://en.wikipedia.org/wiki/Malaria \\
\hline Heart Disease & http://en.wikipedia.org/wiki/Cardiovascular_disease \\
\hline Depression & http://en.wikipedia.org/wiki/Major_depressive_disorder \\
\hline Mal de Debarquement & https://en.wikipedia.org/wiki/Mal_de_debarquement \\
\hline
\end{tabular}


Table 2. Metrics of health-related websites. Analysis of the characteristics of maritime health websites (grey column) and the two control types of websites (dental health and otorhinolaryngology [ORL]/head and neck surgery). Data for the first five categories represent the percentage of occurrence of the feature in the websites, and data for the last two categories represent the means \pm SEM for all the websites of each type

\begin{tabular}{llll}
\hline & Maritime health & Dental health & ORL \\
\hline Health-related information & $25 \%$ & $100 \%$ & $75 \%$ \\
Health information accessible from the homepage & $25 \%$ & $100 \%$ & $50 \%$ \\
Links to other health sites & $75 \%$ & $100 \%$ & $75 \%$ \\
Downloadable resources & $25 \%$ & $75 \%$ & $25 \%$ \\
Presence on social media & $25 \%$ & $100 \%$ & $75 \%$ \\
Overall health information quality & $0.5 \pm 0.5$ & $2.5 \pm 0.5$ & $1.5 \pm 0.6$ \\
Maximal actionability & $15 \pm 15$ & $65 \pm 23.6$ & $30 \pm 5.8$
\end{tabular}

further explorations of the website were required to locate these pages; 3) the presence of links to health information sites (as defined as sites providing medical information for patients - if the sites were not providing direct information, they were not counted); 4) the presence of downloadable resources for patients (e.g., downloadable fact sheets); 5) a social media presence (defined as links to social media accounts displayed on the website); 6) an index of the overall quality of the presented medical information (0: no medical information; 1: general and not specific medical information, just a mention of diseases, no practical information; 2: detailed medical information, in-depth explanation of the diseases, practical information; 3: complete medical information, detailed information complemented with statistics, patient-oriented presentation of critical studies); and finally 7) for each site, a maximal actionability was computed using the "actionability" subscale of the Patient Education Materials Assessment Tool (PEMAT, see below), scored for each item on the best element provided on the website (whatever the page in the site). This last measure was taken as an overall evaluation of the global potential for actionability of the site in terms of health advices to the seafaring population. For the first five aforementioned variables (presence of health-related information, whether health information were accessible from the homepage, presence of links to other health sites, presence of downloadable resources, and presence on social media), a percentage of occurrence was calculated across the four websites. For the last two variables (overall health information quality, and maximal actionability), means and standard error of the mean (SEM) were calculated. All metrics were presented in Table 2 .

While of potential interest, downloadable materials were not specifically analysed in this study. Indeed, since the most consulted online information are the most proximal information in terms of distance (clicks to reach the information), downloadable materials are usually less consulted than main pages or Wikipedia articles. Therefore, primary material was preferred in our analyses over downloadable material. Furthermore, due to the variability of the material which can be used in this way (graphs, fact sheets, or textual files), direct comparisons between items of this category would be impossible to perform.

\section{READABILITY ASSESSMENT AND CONTENT EVALUATION}

Numerous tools are available to assess the degree of readability of a text $[4,10,11]$. Most studies tend to use several of them in order to optimise the analyses [4-6, 12]. In order to assess the readability of the selected Wikipedia's articles, we used a battery of four tests selected among the most commonly used readability analysis tools in the biomedical field, and which has been validated to evaluate readability in the context of online materials [12]. This battery consisted of the Flesch Reading Ease (FRE), the Flesch Kincaid Grade Level (FKGL), the Simple measure of Gobbledygook (SMOG), and the Gunning Fog Index (GFI). All of these tests are considered as highly reliable in readability assessment in a biomedical context [11]. Scores with the FRE are on a scale ranging from 0 to 100,100 being the most readable, while the scores of the three other scales reflects the number of years of education (the level recommended as optimal by the National Institutes of Health $(\mathrm{NIH})$ for communication of health related information to the general public being between 6 and 8 ). As recommended by the $\mathrm{NIH}[13,14]$, all the retrieved texts were formatted for readability assessment purposes: all references, abbreviations, bullets, hyperlinks, colons, semi-colons and hyphens were excluded to insure accurate scoring. All analyses were performed on the first 400 words of the article (adjusted to the closest sentence).

The PEMAT for printable material was used to evaluate the understandability (how easily the presented material can be understood by patients) and the actionability (how the presented material can be effectively used by patients) 
of the articles surveyed [15]. Analysis with the PEMAT generates two final scores (one for understandability and one for actionability) on 100 points scales. For each scores, an average was made between the ratings of two independent judges. Both judges were experienced in using the PEMAT to evaluate online resources, and were qualified in biomedical sciences (holding either a PhD or a MD). While averaged scored were used, individual ratings of the two judges were similar (not significantly different for the two judges, $p=0.88$, Mann-Whitney $U$ test). When appropriate, results were presented as mean \pm SEM.

\section{RESULTS}

\section{WEBSITES CONTENT ANALYSIS}

Websites specifically related to health concerns of seafarers, or maritime medicine in general, were difficult to locate when compared to other medical subjects. With the notable exception of the International Transport Workers' Federation Seafarer website, which was the most complete maritime health site in the sample and provided the most actionable recommendations to seafarers, maritime health websites scored relatively poorly. Indeed, for most of the seven criterions measured, maritime health sites scored lower than other medical sites (Table 2). This was true both for quantitative evaluations (presence of health-related information: maritime health: $25 \%$, dental health: $100 \%$, otorhinolaryngology [ORL]: $75 \%$; accessibility of health information directly from the homepage: maritime health: $25 \%$, dental health: $100 \%$, ORL: $50 \%$ ), as well as for the measures of usability (overall health information quality: maritime health: $0.5 \pm 0.5$, dental health: $2.5 \pm 0.5$, ORL: $1.5 \pm 0.6$; maximal actionability: maritime health: $15 \pm 15$, dental health: $65 \pm 23.6$, ORL: $30 \pm 5.8$, Table 2). Maritime health websites also displayed a lower presence on social media ( $25 \%$ vs. $100 \%$ for dental health and $75 \%$ for ORL). However, the presence of links to other health sites was similar for maritime health and other health fields (maritime health: 75\%, dental health: 100\%, ORL: $75 \%$, Table 2 ). While low, the availability of downloadable resources was comparable for maritime health and other health fields (maritime health: $25 \%$, dental health: $75 \%$, ORL: $25 \%$, Table 2). Typically, downloadable materials were not immediately accessible (distance of two clicks or more from the main webpage), confirming that they were not to be considered as primary information support, but more as supplementary elements for people digging in the sites. Nonetheless, various downloadable materials were available through some distance via the maritime health websites considered (for instance, the Textbook of Maritime Medicine, available at http://textbook.ncmm.no).

\section{TEXTUAL CONTENT ANALYSIS}

The readability of the disorder-related articles was way above what was expected from the lay population, as evaluated both by the FRE $(30.95 \pm 1.88)$ and by the other readability scales (FKGL: $14.05 \pm 0.35$, SMOG: $12.25 \pm 0.31$, GFI: $16.75 \pm 0.58$ ). When evaluated with the PEMAT, both the understandability $(33.42 \pm 2.26)$ and actionability $(5 \pm 5)$ of the articles surveyed were extremely low, making them not adapted as patient education materials. While the pathologies surveyed in the articles could affect the general population (and not solely seafarers), being a seafarer represented a supplementary risk factor for these disorders. However, this particular risk factor was almost never mentioned (the words "seafarer" and "sailor" were absent from the four articles).

\section{DISCUSSION}

The main result of this exploratory study aiming to document the status of health-related online resources available for seafarers, is that the amount of online information on maritime-specific health issues easily available for seafarers and their relatives is extremely small. Furthermore, the few available materials appear to not be fully adapted for the specific needs of the seafaring population.

Despite the fact that seafarers are recognised as being a highly vulnerable population when it comes to health status, very little information were available without extensive search. One could argue that some of the websites in the maritime health sample were not specifically targeted primarily at patients, but at health professionals. However, all of the websites used as control (dental health and otorhinolaryngology) were also targeted at health professionals, and did perform better in terms of conveying useful information to patients. While the relatively low level of understandability of medical websites for lay public have been already noted [4-6], this situation appears to be worse in the case of maritime health.

Several elements might contribute to explain this situation. The first element is undoubtedly the fact that the actual number of health specialists dedicated to maritime health is extremely limited compared to other onshore medical specialties. Thus, the discipline and the health community simply lack the critical mass necessary to efficiently broadcast health-related information on virtual, Internet-supported media. This is particularly important in the case of health portals which are inherently dependant to the presence of an active supporting health community in order to fulfil their goal - something impossible to achieve given the very limited number of health personal dedicated to the field of maritime medicine compared to other medical specialties. One of the factors explaining this situation is probably the lack of a medical specialty specifi- 
cally dedicated to maritime health. Indeed, while specialties which may be relevant for maritime health, such as occupational medicine or preventive medicine, benefit from an official status, maritime medicine itself is not considered as a medical speciality neither in North America (as defined by the American Board of Medical Specialties and by the Royal College of Physicians and Surgeons of Canada), nor in Europe (as defined in the list of medical specialties recognised in the European Union and European Economic Area) - the situation being actually the same worldwide. Despite some efforts in standardisation promoted notably by supra-national organisations, this results not only to important divergences in how maritime medicine is practiced across countries $[2,16]$, but also to the fact that self-identification as a "maritime health specialist" can be difficult to achieve for individuals, leading to a lack of centralisation and consultation in the strategies used to publicise maritime health-related information. The vastly international context in which maritime medicine takes place does not either favour concerted actions [2]. Given what was observed in the present study on the English Internet, it is quite reasonable to predict that the actual situation is even worse for seafarers seeking information in other languages. In other words, the seafarers not reading English would be left with even less available online resources.

In this view, the case of Wikipedia is of interest. While in popular culture, the use of Wikipedia is often associated with students - something actually confirmed by the fact that a majority of students across various fields, including medicine, regularly use Wikipedia for class-related works $[17,18]$, the heavy use of Wikipedia as a knowledge resource is a wide-spread practice on the Internet [3, 19]. Particularly, patients have been demonstrated to use more and more the new media to find information or advices regarding their medical status or the different treatment options [20]. Therefore, and although this still has to be formally documented, it is reasonable to assume that Wikipedia also serves as a primary knowledge source for a lot of seafarers and their relatives when it comes to questions related to their health status.

It is extremely important to remind here that Wikipedia is not supposed to be a patient education portal; the aim of Wikipedia is not to provide practical health-related advices but to realise a summary of human knowledge in an encyclopaedic format. Furthermore, while seafarers were usually not mentioned in the Wikipedia pages observed, the diseases described in these pages encompassed population way larger than just the seafarers, being a seafarer only representing a supplementary risk factor. Nonetheless, due to its exposure and its prominence as an information search portal, the fact that this resource was - once again legitimately - not adapted to seafarers' needs made a possible compensation with this online encyclopaedia for the lacks of otherwise available resources impossible.

Obviously, easily accessible online health information would contribute to increase the quality of life of seafarers and their relatives. In addition, this might also have wider economic consequences. Indeed, previous studies have documented the fact that a wider use of telemedicine could considerably reduce the costs associated with maritime medicine [21]. Particularly, telemedical consultations allow for better diagnosis and therefore contribute to limit the most costly medevac or even ship rerouting [21]. But for seafarers not able to rely on constant telemedical support, or not able to benefit from regular medical controls, accurate, complete, and more importantly, easily available medical-related information could contribute to a similar extend to this reduction of health-related costs in maritime industries.

\section{CONCLUSIONS}

While major efforts have been made and are still being made worldwide to increase accessibility to medical care for seafarers and the global level of medical education of seafarers, most of the initiatives so far have been targeted toward punctual actions aiming to provide knowledge in structured forms (ranging from onshore formation and certification of minimum health knowledge requirements, to regulations such as keeping medical handbooks on-board). However, while the impact of such initiatives on seafarer health is highly positive, there is still a major gap between seafarer global health information needs and what is actually available to them. Due to the limited population of maritime health professionals, offering high quality moderated online resources will be difficult to achieve without operative models specifically designed for maritime health. Thus, a lot has still to be made in order to provide the seafaring population with continuous and reliable sources of health-related information outside of the conventional structured education and normative framework.

\section{REFERENCES}

1. Ulven AJ. Medical and psychological challenges in the offshore petroleum industry. Int Marit Health 2009; 60: 40-42.

2. Guitton MJ. Telemedicine at sea and onshore: divergences and convergences. Int Marit Health 2015; 66: 18-21. doi: 10.5603/ IMH.2015.0005.

3. Brossard D. New media landscapes and the science information consumer. P Natl Acad Sci USA 2013; 110: 14096-14101. doi: 10.1073/pnas.1212744110.

4. Eloy JA, Li S, Kasabwala K, Agarwal N, Hansberry DR et al. Readability assessment of patient education materials on major otolaryngology association websites. Otolaryngol Head Neck Surg 2012; 147 : 848-854. doi: 10.1177/0194599812456152.

5. Hansberry DR, Agarwal N, Shah R, Schmitt PJ, Baredes S et al. Analysis of the readability of patient education materials from 
surgical subspecialties. Laryngoscope 2014; 124: 405-412. doi: 10.1002/lary.24261.

6. Alamoudi U, Hong P. Readability and quality assessment of websites related to microtia and aural atresia. Int J Pediatr Otorhinolaryngol 2015; 79: 151-156. doi: 10.1016/j.jporl.2014.11.027.

7. Brewer PA, Barry M. Survey of web-based health care information for prospective cruise line passengers. J Travel Med 2002; 9: 194-197.

8. Grefenstette G, Nioche J. Estimation of English and non-English language use on the WWW. Proceedings of RIAO'2000 "Content -Based Multimedia Information Access", Paris, 12-14 April 2000, pp. 237-246. http://arxiv.org/abs/cs/0006032.

9. Clément M, Guitton MJ. Interacting with bots online: Users's reaction to actions of automated programs in Wikipedia. Comput Human Behav 2015; 50: 66-75. doi: 10.1016/j.chb.2015.03.078.

10. Meade CD, Smith CF. Readability formulas: cautions and criteria. Patient Educ Couns 1991; 17: 153-158.

11. Wang LW, Miller M. Assessing readability formula differences with written health information materials: application, results, and recommendations. Research in Social and Administrative Pharmacy 2013; 9: 503-516. doi: 10.1016/j.sapharm.2012.05.009.

12. Guitton MJ. Swimming with mermaids: communication and social density in the Second Life merfolk community. Comput Human Behav 2015; 48: 226-235. doi: 10.1016/j.chb.2015.02.004.

13. National Institutes of Health. How to write easy to read health materials. http://www.nlm.nih.gov/medlineplus/etr.html. Accessed: January 6, 2015.
14. Friedman DB, Hoffman-Goetz L. A systematic review of readability and comprehension instruments used for print and web-based cancer information. Health Education Behavior 2006; 33: 352-373. doi: 10.1177/1090198105277329.

15. Shoemaker SJ, Wolf MS, Brach C. Development of the Patient Education Materials Assessment Tool (PEMAT): a new measure of understandability and actionability for print and audiovisual patient information. Patient Educ Couns 2014; 96: 395-403. doi: 10.1016/j.pec.2014.05.027.

16. Ricci G, Pirillo I, Rinuncini C, Amenta F. Medical assistance at sea: legal and medico-legal problems. Int Marit Health 2014; 65 : 205-209. doi: 10.5603/IMH.2014.0039.

17. Judd T, Kennedy G. Expediency-based practice? Medical students' reliance on Google and Wikipedia for biomedical inquiries. Br J Educ Technol 2011; 42: 351-360. doi: 10.1111/ j.1467-8535.2009.01019.x.

18. Knight C, Pryke S (2012) Wikipedia and the University, a case study. Teach High Educ 2012; 17: 649-659. doi: 10.1080/ 13562517.2012.666734.

19. Fallis D. Toward an epistemology of Wikipedia. J Am Soc Inf Sci Tec 2008; 59: 1662-1674. doi: 10.1002/asi/20870.

20. Laurent, MR, Vickers TJ. Seeking health information online: does Wikipedia matter? J Am Med Inform Assn 2009; 16: 471-479. doi: 10.1197/jamia.M3059.

21. Henny C, Hartington K, Scott S, Tveiten A, Canals L. The business case for telemedicine. Int Marit Health 2013; 64: 129-135. 